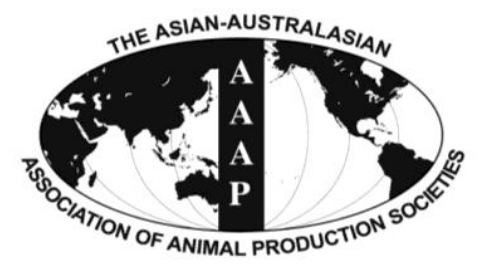

\title{
Expression Profiling of WSSV ORF 199 and Shrimp Ubiquitin Conjugating Enzyme in WSSV Infected Penaeus monodon
}

\author{
K. Jeena, K. Pani Prasad*, Mujahid Khan Pathan ${ }^{1}$ and P. Gireesh Babu ${ }^{1}$ \\ Aquatic Environment and Health Management Division, Central Institute of Fisheries Education, \\ Mumbai-400061, India
}

\begin{abstract}
White spot syndrome virus (WSSV) is one of the major viral pathogens affecting shrimp aquaculture. Four proteins, WSSV199, WSSV 222, WSSV 249 and WSSV 403, from WSSV are predicted to encode a RING-H2 domain, which in presence of ubiquitin conjugating enzyme (E2) in shrimp can function as viral E3 ligase and modulate the host ubiquitin proteasome pathway. Modulation of host ubiquitin proteasome pathway by viral proteins is implicated in viral pathogenesis. In the present study, a time course expression profile analysis of WSSV Open Reading Frame (ORF) 199 and Penaeus monodon ubiquitin conjugating enzyme (PmUbc) was carried out at 0, 3, 6, 12, 24, 48 and $72 \mathrm{~h}$ post WSSV challenge by semi-quantitative RT-PCR as well as Real Time PCR. EF1 $\alpha$ was used as reference control to normalize the expression levels. A significant increase in PmUbc expression at $24 \mathrm{~h}$ post infection (h.p.i) was observed followed by a decline till 72 h.p.i. Expression of WSSV199 was observed at 24 h.p.i in WSSV infected P. monodon. Since the up-regulation of PmUbc was observed at 24 h.p.i where WSSV199 expression was detected, it can be speculated that these proteins might interact with host ubiquitination pathway for viral pathogenesis. However, further studies need to be carried out to unfold the molecular mechanism of interaction between host and virus to devise efficient control strategies for this chaos in the shrimp culture industry. (Key Words: WSSV 199, Shrimp, Ubiquitin Conjugating Enzyme, Penaeus monodon)
\end{abstract}

\section{INTRODUCTION}

The white spot syndrome virus (WSSV) has the greatest impact in shrimp aquaculture, both in terms of infectivity and productivity. It is a rapidly replicating and extremely virulent shrimp pathogen infecting Penaeid shrimp and has emerged globally as one of the most prevalent and widespread (Arturo, 2010). It causes up to $100 \%$ mortality within 3 to 10 days of infection resulting in huge economic losses to shrimp farming industry (Kimura et al., 2000) and is generally characterized by white spots in the exomesoderm under the carapace. It has a circular dsDNA genome about $300 \mathrm{kbp}$ that contains approximately 185 open reading frames (ORFs) (Yang et al., 2001; Hulten et al., 2001). It is one of the largest viral genomes. WSSV was first detected in Asia, with occurrence in Taiwan (Chou et al., 1995), Japan (Nakano et al., 1994) and Korea (Park et

\footnotetext{
* Corresponding Author: K. Pani Prasad. Tel: +91-22-26320451, Fax: +91-22-26361573, E-mail: pprasad35@gmail.com

${ }^{1}$ Fish Genetics and Biotechnology Division, Central Institute of Fisheries Education, Mumbai, 400061 India. Submitted Dec. 8, 2011; Accepted Mar. 23, 2012; Revised Apr. 23, 2012
}

al., 1998) and now is widespread across the world.

There is no cure against this devastating virus of shrimp and this could be attributed to limitation of expertise with regard to shrimp immune system. Understanding the mechanism of interaction between the host proteins and viral proteins at molecular level would greatly help us in formulating effective therapeutic strategies. Ubiquitination is a well studied proteasome pathway and plays key roles in viral infection and facilitates activities required for various aspects of virus life cycle, from entry (Galinier et al., 2002) through replication (Everett et al., 1999; Parkinson and Everett, 2001) and enhanced cell survival (Thomas et al., 1999; Winberg et al., 2000) to viral release (Harty et al., 2001; Yasuda et al., 2002). Ubiquitin conjugating enzyme is one of the active components in the pathway of ubiquitin mediated protein degradation and has been found to possess important role in WSS viral pathogenesis. The ubiquitin proteasome pathway degrades abnormal and short lived proteins (Laney and Hochstrasser, 1999). In the ubiquitin dependant proteolytic pathway, ubiquitin is linked to substrates through a well organized process involving the sequential action of a ubiquitin- activating enzyme (E1), a 
ubiquitin- conjugating enzyme (E2) and, a ubiquitin ligase (E3). Polyubiquitinated proteins are then targeted to the 26S proteasome for degradation (Hershko and Ciechanover, 1998; Deshaies, 1999; Ciechanover et al., 2000; Pickart, 2001).

Viruses have evolved to use cellular pathways to their advantage including the ubiquitin proteasome pathway of protein degradation. In most of the cases viruses do produce proteins that hijack cellular E3 ligases to modify their substrate specificity to get rid of nonessential cellular proteins, in particular inhibitors of the cell cycle. They are also known to inhibit E3 ligases to retard specific protein degradation or even use the system to control the level of expression of their native proteins (Blanchette and Branton, 2009). The involvement of RING finger domain, by acting as E3 ubiquitin protein ligase, in specific ubiquitination events was revealed in a study by Freemont, 2000. RING finger proteins in other cellular or viral systems may function as a ubiquitin ligase, E3 (Hershko and Ciechanover, 1998; Yamao, 1999; Ciechanover, 2003; Wang et al., 2005; He et al., 2006). As such four proteins of WSSV like WSSV 199, WSSV 222, WSSV 249 and WSSV 403 are reported to carry a common feature, the RING $\mathrm{H} 2$ domain, and the ubiquitination activity has been demonstrated for three of them (WSV222, WSV249, WSV 403), (Wang et al., 2005; He et al., 2006; Fang and Kwang, 2008) with one of the important enzyme ubiquitin ligase of the ubiquitin proteasome pathway while no information is available regarding WSSV 199 . Hence a study was conducted on the expression profiles of the ubiquitin conjugating enzyme and one of RING finger domain containing proteins of WSSV i.e. WSSV 199 in WSSV infected shrimp, Penaeus monodon.

\section{MATERIAL AND METHODS}

\section{Shrimp rearing}

P. monodon of $15 \pm 2 \mathrm{~g}$ size were transported from Pancham Aqua Farm, Maharashtra, India and maintained in 1,000 L FRP tanks (25 shrimp/tank) in natural seawater of $35 \mathrm{ppt}$ with continuous aeration. The shrimp were fed ad libitum with artificial pelleted feed (CP feeds) twice a day. Left over feed was siphoned daily and $30 \%$ water exchange was done once in a week. Salinity was maintained at $35 \mathrm{ppt}$, temperature 22 to $25^{\circ} \mathrm{C}$ and $\mathrm{pH} 7.8$, throughout the experimental period and the health condition of the animals was monitored regularly. Shrimps were held for a minimum of 2 weeks prior to experimental use and feeding was stopped $24 \mathrm{~h}$ before treatment.

\section{Preparation of viral inoculum}

WSSV infected $P$. monodon with prominent white spots were collected and head soft tissues from shrimp were homogenized in chilled sterile 1X Phosphate Buffered Saline (PBS) $\mathrm{pH}$ 7.4. The homogenate was centrifuged at $2,460 \mathrm{~g}$ for $20 \mathrm{~min}$ at $4^{\circ} \mathrm{C}$. The supernatant was again subjected to centrifugation to remove the cell debris. The supernatant was filtered $(0.22 \mu$-pore diameter-filter). The presence of the viral particles in the inoculum was confirmed with WSSV detection kit (Bangalore Genei, India) following manufacturer's instructions. Infectivity of the viral inoculum was confirmed by performing in vivo titration with healthy shrimp. The viral dose that caused 100 percent mortality in $96 \mathrm{~h}$ was used for time course analysis.

\section{Experimental conditions}

Four groups of 12 shrimps $(15 \pm 2 \mathrm{~g})$ each were maintained in plastic crates of $25 \mathrm{~L}$ capacity with adequate aeration. After acclimatization for $3 \mathrm{~d}$, three groups of shrimps were injected with $100 \mu$ of WSSV inoculum ventral to the second abdominal segment. The unchallenged fourth group served as control. Six time points after challenging i.e., $0,3,6,12,24,48$ and $72 \mathrm{~h}$ was selected to quantify the ubiquitin conjugating enzyme gene as well as the WSSV 199 genes in comparison to unchallenged control. For this four shrimps were collected at each time interval from all groups including control.

\section{RNA extraction and cDNA synthesis}

Total RNA from muscle tissue of infected and control shrimp at different time points were isolated using TRIzol reagent (Invitrogen, USA) based on manufacturers' instructions. The total RNA was quantified by measuring absorbance at $260 \mathrm{~nm}$ in a UV Biophotometer (Eppendorf AG, Germany) and quality was checked on a $1 \%$ agarose gel. Total RNA was treated with RNase free DNase 1 (Fermentas, USA) to remove DNA contamination. Firststrand cDNA synthesis was carried out using $2 \mu \mathrm{g}$ of DNase-treated total RNA as template. Reverse transcription was performed using Moloney leukemia virus reverse transcriptase (Fermentas, USA), $0.5 \mu \mathrm{g}$ of oligo $(\mathrm{dT})_{18}$ primer, $1 \times$ RT reaction buffer, $1 \mathrm{mM}$ each of dNTPs, $20 \mathrm{U}$ of ribonuclease inhibitor and 40 units of reverse transcriptase in a final reaction volume of $20 \mu \mathrm{l}$. The reaction was carried as per the manufacturers' instructions.

\section{Semi quantitative RT-PCR analysis}

Semi quantitative RT-PCR was performed using $50 \mu \mathrm{g}$ cDNA as template. The primers 199-5 (5'-TTCAACCAA ATGGGCAAGCTC-3') and 199-3 (5'- CGTTGTGGAA GCAATGACCG -3') were used to amplify WSSV 199 and primers PmUbc-5 (5'-TCAAAGGCACTCAGCACCAGTG3') and PmUbc-3 (5'-TCATACACGGACCCAGGTGG-3') were used to amplify PmUbc, to generate $150 \mathrm{bp}$ fragments of WSSV 199 and PmUbc. EF1- $\alpha$ was used as internal 
control and was amplified with the primers EF1- $\alpha$ forward (5'-GGTGCTGGACAAGCTGAAGGC -3') and EF1- $\alpha$ reverse (5'-CGTTCCGGTGATCATGTTCTTGATG-3') primer pair because its concentrations were found to be unaffected across the treatments compared to beta actin as determined by a pilot study. The thermocycling parameters consisted of an initial denaturation at $94^{\circ} \mathrm{C}$ for $3 \mathrm{~min}$ followed by 30 cycles of $94^{\circ} \mathrm{C}$ for $15 \mathrm{~s}, 60^{\circ} \mathrm{C}$ for $20 \mathrm{~s}$ and $72^{\circ} \mathrm{C}$ for $20 \mathrm{~s}$. The final extension was done for $5 \mathrm{~min}$ at $72^{\circ} \mathrm{C}$. Hold temperature was set at $4^{\circ} \mathrm{C}$. The PCR products were run on $2 \%$ gels for further analysis. PCR products were quantified using Gene Tools Software.

\section{Real time PCR analysis}

Real-time PCR amplifications were carried out in ABI 7500 Real Time PCR detection system (Applied Biosystems, USA) using SYBR Green (Fermentas, USA) chemistry. The same primer pairs used in Semi-quantitative RT-PCR were used to amplify both WSSV 199 and PmUbc. The thermal profile used for PCR amplification consisted of initial denaturation at $95^{\circ} \mathrm{C}$ for $10 \mathrm{~min}$, followed by 40 cycles of denaturation at $95^{\circ} \mathrm{C}$ for $15 \mathrm{~s}$, annealing and extension at $60^{\circ} \mathrm{C}$ for $1 \mathrm{~min}$. Melting curve analysis of amplification products were performed at the end of each PCR reaction to confirm that only one PCR product was amplified and detected. Comparative CT method was used to estimate the relative expression of the target mRNA. Briefly, $\Delta \mathrm{CT}$ was calculated by subtracting CT value of internal control from target gene and then mean $\Delta \mathrm{CT}$ was calculated from this normalized $\Delta \mathrm{CT}$ value. $\Delta \Delta \mathrm{CT}$ was calculated with respect to control by, subtracting mean $\Delta \mathrm{CT}$ of control treatment from mean $\Delta \mathrm{CT}$ of the target gene. Fold change at various time intervals was calculated by $2^{-\Delta \Lambda}$ CT. EF1 $\alpha$ was selected as internal control for normalization.

\section{Statistical analysis}

The differences in transcript levels between different time intervals were tested for statistical significance using one-way ANOVA followed by Duncan's new multiple range test using the statistical package, (SAS Inc., USA) (Attwood et al., 2007; Alenjandro et al., 2008) p value below 0.05 was considered statistically significant. The results were expressed as the mean $\pm \mathrm{SD}$.

\section{RESULTS}

Semi quantitative analysis of WSSV 199 in time course study

The expression pattern of WSSV199 in WSSV infected shrimps was investigated in a time course study. The expression pattern varied between different time intervals.

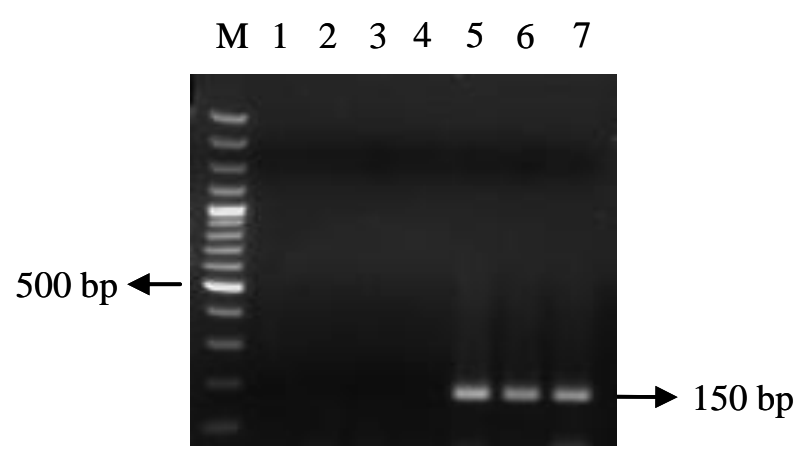

Figure 1. WSSV 199 expression at different time points post infection.

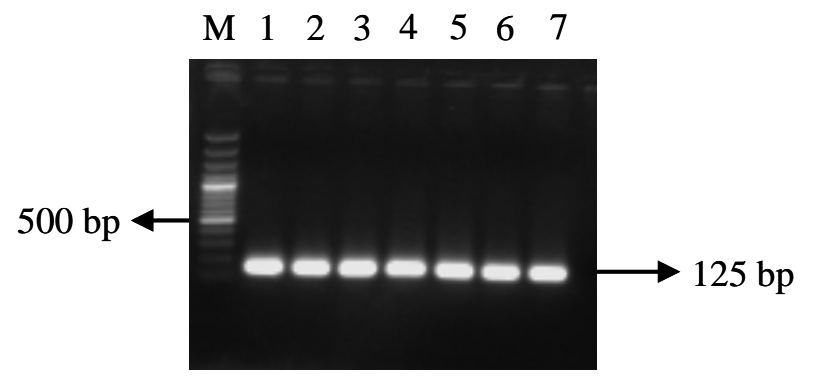

Figure 2. EF1- $\alpha$ expression. Lane M: 100 bp plus marker, lane 1-7: h post injection, $0,3,6,12,24,48$ and 72 h.p.i, respectively.

Expression was not observed in RT-PCR analysis in 0 to 12 h.p.i while expression was observed at 24 h.p.i. The expression showed an increasing trend from 24 to 72 h.p.i. (Figure 1). The expression pattern was compared with that of EF1- $\alpha$, whose expression remained unchanged throughout the time period (Figure 2).

\section{Semi quantitative analysis of PmUbc in time course study}

RT-PCR analysis of PmUbc showed the expression pattern in different time points post WSSV infection. In the case of PmUbc, the expression was seen to be more or less similar till 12 h.p.i while a sudden increase in transcript

\section{$\begin{array}{llllllll}M & 1 & 2 & 3 & 4 & 5 & 6 & 7\end{array}$}

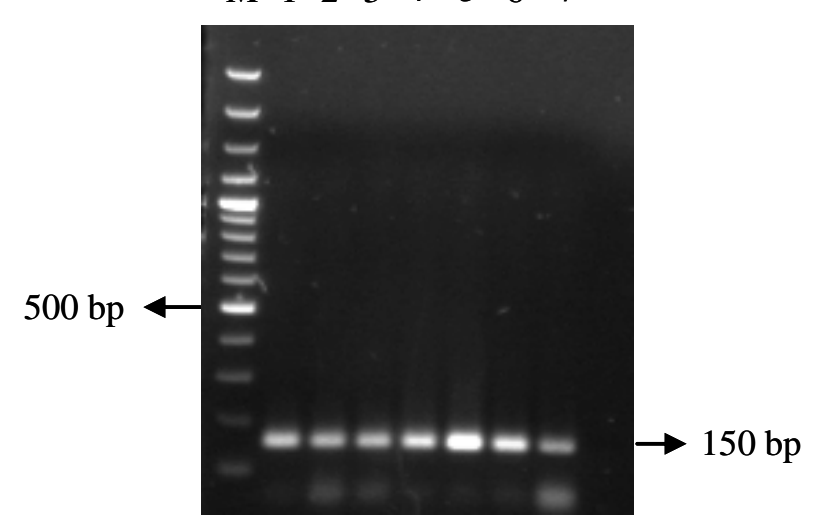

Figure 3. Expression of PmUbc in different time points post wssv infection. 


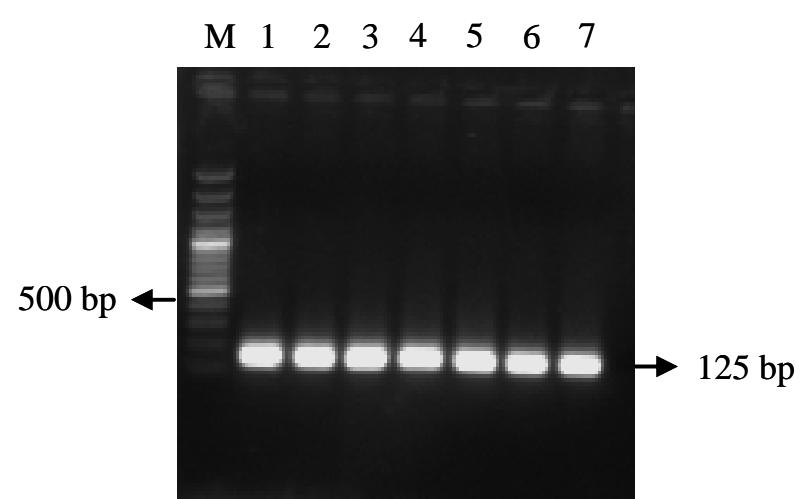

Figure 4. EF1- $\alpha$ expression throughout the time period. Lane M: $100 \mathrm{bp}$ plus marker, lane 1-7: h post injection, $0,3,6,12,24,48$ and 72 h.p.i, respectively.

level was observed at 24 h.p.i followed by gradual decrease till 72 h.p.i (Figure 3). The internal control EF1- $\alpha$ expression was found to be constant throughout the time period (Figure 4).

Real time expression profiles of WSSV 199 and PmUbc in muscle tissue of WSSV infected shrimp

Relative expression analysis of the PmUbc and WSSV199 genes at different time points post WSSV infection was also studied by Real Time RT-PCR using SYBR green chemistry. EF1- $\alpha$ was used as the internal control. In Real time analysis it was observed that there was a tremendous increase in the expression of PmUbc at 24 h.p.i. followed by a drastic decrease at 48 h.p.i (Figure 5). A change of greater than 12 fold was observed in the expression of PmUbc at 24 h.p.i. and then showed a gradual decrease. The WSSV 199 was also found to express at 24 h.p.i., peaked at 48 h.p.i. and shown a decline at 72 h.p.i. (Figure 6). A fold change of greater than 7,000 was observed in the expression of WSSV 199 at 24 h.p.i. The expression of EF1- $\alpha$ remained same throughout the experimental period. Melt curve analysis of the genes

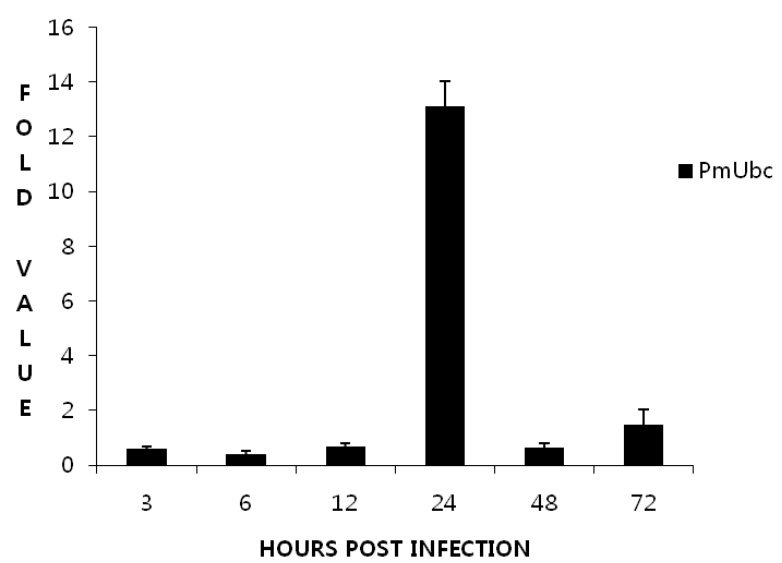

Figure 5. Fold changes (mean $\pm \mathrm{SD}$ ) in expression of PmUbc in real time-PCR.

showed a single peak for all the genes studied and expression levels were found to be statistically significant.

\section{DISCUSSION}

Four proteins of WSSV, namely WSSV199, WSSV222, WSSV249 and WSSV403 are known to contain RING-H2 domains (Yang et al., 2001; Wang et al., 2005). Many RING finger domains simultaneously bind ubiquitination enzymes and their substrates and hence function as ligases. Therefore, it is known that viral proteins containing RING finger domain might play a key role in the modification of host's ubiquitination pathway. Among these, WSSV222 is shown to mediate the degradation of a shrimp tumor suppressor as a viral E3 ligase (He et al., 2006). Fang and Kwang (2008) have reported that WSSV403 regulates the latency state of WSSV by virtue of its E3 ligase function. The WSSV249, also acting as an E3 ligase is reported to sequester the E2 ubiquitin-conjugating enzyme in Litopenaeus vannamei (Wang et al., 2005). As no reports were found on the functional aspects of WSSV199, the present study was designed to check whether the WSSV199 and shrimp

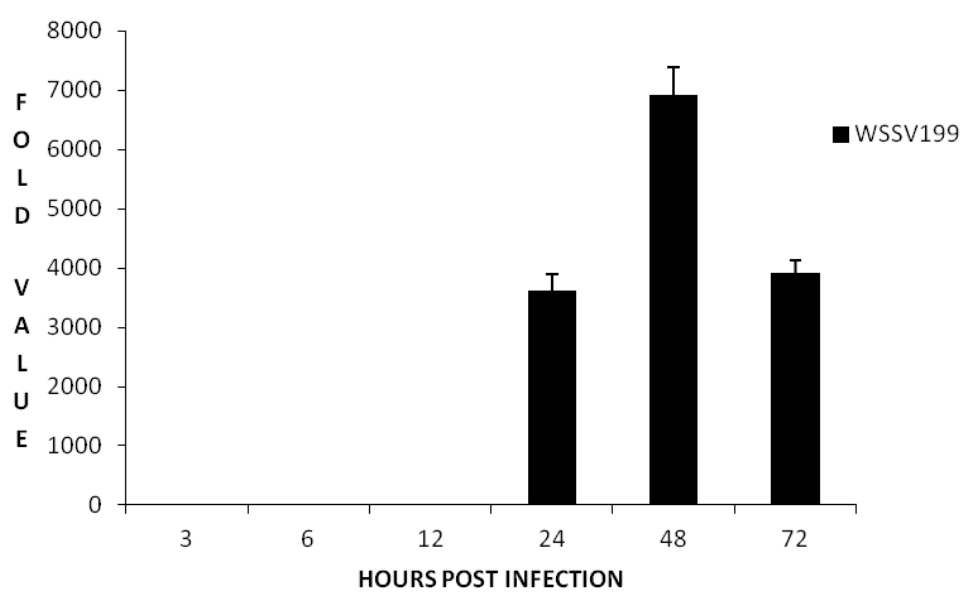

Figure 6. Fold changes (mean \pm SD) in expression of WSSV 199 in real time-PCR. 
ubiquitin conjugating enzyme gene expression profiles in WSSV challenged $P$. monodon corresponds to the observations made in $L$. vannamei with respect to WSSV249 and others. The expression levels of PmUbc and WSSV 199 was quantified by normalizing with the expression of EF1- $\alpha$.

The PmUbc expression was observed at all the time points tested including the control i.e., the unchallenged group. The expression was almost constant till 12 h.p.i. while a significant increase was observed at $24 \mathrm{~h}$ post infection (h.p.i). The expression again declined from 48 to 72 h.p.i. A similar study by Wang et al. (2005) in $L$. vannamei revealed that the expression of ubiquitin conjugating enzyme (PvUbC) in WSSV infected shrimp increases consistently with time while it shows very weak expression in healthy shrimp. They have suggested that PvUbC expression is induced by WSSV infection. However, the variation in the expression pattern of PmUbc in comparison to PvUbC as observed in the present study may be due to the differences at species level. Also, Wang et al. (2005) studied the expression pattern till 48 h.p.i while in the present study the expression was studied till 72 h.p.i. The ubiquitin conjugating enzyme expression is seen not only in the infected animal but also in the normal healthy animal. This is quite expected because ubiquitin-dependent proteolysis regulates protein abundance and serves as a central regulatory function in many biological processes such as cell cycle regulation, signal transduction, transcriptional regulation, DNA repair, inflammatory response, and antigen presentation in eukaryotic cells (Hershko et al., 1980; Haas and Rose, 1981).Time course expression analysis of WSSV199 at different time points in $P$. monodon post WSSV challenge revealed that the gene expression was maximum at 24 h.p.i and its more than 7,000 folds when compared to 0 h.p.i. On comparison with the expression profile of PmUbc it can be said that the upregulation in PmUbc expression might be correlated with the expression of WSSV199 at 24 h.p.i. Subsequent decrease in PmUbc expression at 48 h.p.i. could be because the WSSV199 proteins modulate its expression for efficient pathogenesis. It can be speculated from the above observations that the host machinery tries to defend the viral multiplication by ubiquitin mediated protein degradation, which is evident with an increase in PmUbc expression at 24 h.p.i. However, soon after the WSSV199 protein modulate the expression of PmUbc for viral pathogenesis, a decline in PmUbc expression was observed from 48 h.p.i onwards. This is supported by Wang et al. (2005) who reported that the RING-H2 protein WSSV249 from WSSV may function as an E3 ligase by sequestering PvUbc for viral pathogenesis in shrimp (Wang et al., 2005). Many findings have shown that viral infection can up- regulate expression of ubiquitin (Latchman et al., 1987; Kemp and Latchman, 1988), suggesting that the ubiquitin system may play a key role in the course of viral infection. Increasing number of RFPs (RING Finger Proteins) has been identified in viruses. For example, the $i e 2$ gene of Autographa californica nuclear polyhedrosis virus (ACNPV) encodes for an RFP IE2 protein, which appears to block the cell cycle progression in S phase (Prikhod'ko and Miller, 1999). IE2 and PE38 of Bombyx mori Nucleopolyhedrovirus function as E3, and may transregulate the viral transcription and DNA replication (Imai et al., 2003). Recently, several plant homeodomaincontaining viral proteins have been identified as E3 ubiquitin ligases which promote immune evasion by down regulating proteins that govern immune recognition (Coscoy and Ganem, 2003).

Lorick et al. (1999) reported that a large number of RING finger-containing proteins, with otherwise diverse structures and functions, may play previously unappreciated roles in modulating protein levels via ubiquitination. In the presence of an E2 conjugating enzyme from shrimp, WSSV403 can ubiquitinate itself in vitro, indicating it can function as a viral E3 ligase. WSSV403 is a regulator of latency state of WSSV by virtue of its E3 ligase function (He and Kwang, 2008).

The present study reveals novel report on the expression profile of WSSV199 and the expression patterns of PmUbc at transcript level in WSSV infected P. monodon. WSSV199 is predicted to encode a RING $\mathrm{H} 2$ domain and its expression patterns observed in the current study reveals the importance of those genes in WSSV pathogenesis by virtue of their role as viral E3 ligase. However, further studies need to be undertaken to unfold the molecular mechanism of these virus host interactions to be able to design an effective therapy against this dreaded disease of shrimp.

\section{ACKNOWLEDGEMENTS}

The authors acknowledge Dr. W. S. Lakra, Director/ Vice Chancellor, CIFE, Mumbai, India for providing all facilities and Indian Council of Agricultural Research, New Delhi, India for their financial support.

\section{REFERENCES}

Arturo. 2010. White spot syndrome virus: an overview on an emergent concern. Vet. Res. 41:43.

Attwood, T. S., Jason L Blum, Kevin J Kroll, Vishal Patel, Detlef Birkholz, Nancy J Szabo, Suzanne Z Fisher, Robert McKenna, Martha Campbell-Thompson and Nancy D Denslow. 2007. Distinct expression and activity profiles of largemouth bass (Micropterus salmoides) estrogen receptors in response to estradiol and nonylphenol. J. Mol. Endocrinol. 39:223-237. 
Blanchette, P. and E. P. Branton. 2009. Manipulation of the ubiquitin-proteasome pathway by small DNA tumor viruses. Virology 384:317-323.

Chou, H. Y., C. Y. Huang, C. H. Wang, H. C. Chiang and C. F Lo. 1995. Pathogenecity of a Baculovirus infection causing white spot syndrome in cultured penaied shrimp in Taiwan. Dis. Aquat. Org. 23:165-173.

Ciechanover, A. Orian and A. L. Schwartz. 2000. Ubiquitinmediated proteolysis: biological regulation via destruction. Bioessays 22:442-451.

Ciechanover, A. 2003. The ubiquitin proteolytic system and pathogenesis of human diseases: a novel platform for mechanism -based drug targeting. Bio-Chem. Soc. Trans. 31: 474-481.

Coscoy, L. and D. Ganem. 2003. PHD domains and E3 ubiquitin ligases: viruses make the connection. Trends Cell Biol. 13:712.

Deshaies, R. J. 1999. SCF and Cullin/ringH2 based ubiquitin ligases. Annu. Rev. Cell Dev. Biol. 15:435-467.

Everett, R. D., W. C. Earnshaw, J. Findlay and P. Lomonte. 1999. Specific destruction of kinetochore protein CENP-C and disruption of cell division byherpes simplex virus immediateearly protein Vmw110. EMBO J. 18:1526-1538.

Fang, H. and J. Kwang. 2008. Identification and characterization of a new E3 ubiquitin ligase in white spot syndrome virus involved in virus latency. Virology 5:151.

Galinier, R. E., H. Gout, L. J. Jacob, Wood and J. Chroboczek. 2002. Adenovirus protein involved in virus internalization recruits ubiquitin protein ligases. Biochemistry 41:1429914305.

Harty, R. N., M. E. Brown, J. P. Mc Gettigan, G. Wang, H. R. Jayakar, J. M. Huibregtse, M. A. Whitt and M. J. Schnell. 2001. Rhabdoviruses and the cellular ubiquitin-proteosome system: a budding interaction. J. Virol. 75:10623-10629.

Haas, A. L. and I. A. Rose. 1981. Hemin inhibits ATP dependent ubiquitin dependent proteolysis: role of hemin in regulating ubiquitin conjugate degradation. Proc. Natl. Acad. Sci. USA. 78: 6845-6848.

He, F. B., J. Fenner, A. K. Godwin and J. Kwang. 2006. White spot syndrome virus open reading frame 222 encodes a viral E3 ligase and mediates degradation of a host tumour suppressor via ubiquitination. J. Virol. 80:3884-3892.

Hershko, A., A. Ciechanover, H. Heller, A. L. Haas and I. A. Rose. 1980. Proposed role of ATP in protein breakdown conjugation of protein with multiple chains of the polypeptide of ATP dependant proteolysis. Proc. Natl. Acad. Sci. USA. 77:17831786.

Hershko, A. and A. Ciechanover. 1998. The ubiquitin system. Annu. Rev. Biochem. 67:425-479.

Imai, N., N. Matsuda, K. J. Tanaka, A. Nakano, S. Matsumoto and W. K. Kwang. 2003. Ubiquitin ligase activities of Bombyx mori Nucleopolyhedrovirus RING finger proteins. J. Virol. 77:923-930.

Kemp, L. M. and D. S. Latchman. 1988. The herpes simplex virus type I immediate-early protein ICP 4 specifically induces increased transcription of human ubiquitin $\mathrm{B}$ gene without affecting the ubiquitin A and C genes. Virology 166:258-261.
Kimura, N., N. Shimada, M. Fukunda, Y. Ishijima, H. Miyazaki and A. Ishii. 2000. Regulation of cellular functions by nucleoside diphosphate kinases in mammals. J. Bioenerg. Biomembr. 32:309-315.

Laney, J. D. and M. Hochstrasser. 1999. Substrate targeting in the ubiquitin system. Cell 97:427-430.

Latchman, D. S., J. K. Estridge and L. M. Kemp. 1987. Transcriptional induction of the ubiquitin gene during herpes simplex virus infection is dependent upon the viral immediate -early protein, ICP4. Nucleic Acid Res. 15:7283-7293.

Lorick, K. L., J. P. Jensen, S. Fang, A. M. Ong, S. Hatakeyama and A. M. Weissman. 1999. RING fingers mediate ubiquitinconjugating enzyme (E2) dependant ubiquitination. Proc. Natl. Acad. Sci. USA 96:11364-11369.

Nakano, H., H. Koube, S. Umezawa, K. Monoyama, M. Hiraoka, K. Inouye and N. Oseko. 1994. Mass mortalities of cultured Kuruma shrimp, Penaeus japonicus, in Japan in 1993: Epizootiological survey and infection trials. Fish Pathol. 29: 135-139.

Park, J. H., Y. S. Lee, S. Lee and Y. Lee. 1998. An infectious viral disease of penaeid shrimp newly found in Korea. Dis. Aquat.Org. 23:165-173.

Parkinson, J. and R. D. Everett. 2001. Alphaherpesvirus proteins related to herpes simplex virus type 1 ICP0 induce the formation of co localizing, conjugated ubiquitin. J. Virol. 75:5357-5362.

Pickart, C. M. 2001. Mechanisms underlying ubiquitination. Annu. Rev. Biochem. 70:503-533.

Prikhod'ko, E. A. and L. K. Miller. 1998. Role of Baculovirus ie-2 and its RING finger in cell cycle arrest. J. Virol. 72:684-692.

Thomas, M., D. Pim and L. Banks. 1999. The role of the E6-p53 interaction in the molecular pathogenesis of HPV. Oncogene 18:7690-7700.

Van Hulten, M. C., J. Witteveldt, S. Peters, N. Kloosterboer, R. Tarchini and M. Fiers. 2001. The white spot syndrome virus genome sequence. Virology 286:7-22.

Vazquez, A. P., Aswathy Sreedharan and Carol L. Bender. 2010. Transcriptional studies of the hrpM/opgH gene in Pseudomonas syringae during biofilm formation and in response to different environmental challenges. Environ. Microbiol. 12:1452-1467.

Wang, Z., H. K. Chua, A. A. Gusti, F. He, B. Fenner, I. Manopo, H. Wang and J. Kwang. 2005. RING-H2 protein WSSV 249 from white spot syndrome virus sequesters a shrimp ubiquitinconjugating enzyme, PvUbc, for viral pathogenesis. J. Virol. 79:8764-8772.

Winberg, G. L., F. Matskova, P. Chen, D. Plant, G. Rotin, R. Gish, I. Ingham, Ernberg and T. Pawson. 2000. Latent membrane protein 2A of Epstein- Barr virus binds WW domain E3 protein-ubiquitin ligases that ubiquitinated B-cell tyrosine kinases. Mol. Cell. Biol. 20:8526-8535.

Yamao, F. 1999. Ubiquitin system: selectivity and timing of protein destruction. J. Biochem. 125:223-229.

Yang, F., J. He, X. Lin, Q. Li, D. Pan and X. Zhang. 2001. Complete genome sequence of the shrimp white spot bacilliform virus. J. Virol. 75:11811-11820.

Yasuda, J., E. Hunter, M. Nakao and H. Shida. 2002. Functional involvement of a novel Nedd4-like ubiquitin ligase on retrovirus budding. EMBO Rep. 3:636-640. 